\title{
A distinção entre reforçamentos positivo e negativo em livros de ensino de análise do comportamento
}

\author{
The distinction between positive and negative reinforcements in behavior \\ analysis text books
}

\section{La distinción entre refuerzos positivo y negativo en los libros de enseñanza de análisis de la conducta}

Edson Luiz Nascimento dos Santos ${ }^{1}$ e Felipe Lustosa Leite ${ }^{2}$

[1] [2] Universidade Federal do Pará - UFPA I Título abreviado: Distinção entre Reforçamentos Positivo e Negativo | Endereço para correspondência: Email: Edson L. N. dos Santos (edsonsantos_87@hotmail.com); Felipe L. Leite (felipeleite82@gmail.com)

\begin{abstract}
Resumo: Os conceitos de reforçamento positivo e reforçamento negativo têm sido tradicionalmente definidos por meio da apresentação e da remoção de estímulos, respectivamente. Michael (1975) questionou essa distinção, afirmando que em inúmeras situações é difícil distinguir tais processos com base em apresentação e em remoção. Além disso, considerou equivocado o tratamento como sinônimos de estímulos reforçadores negativos e de estímulos aversivos, o que aproximaria reforçamento negativo de punição e criaria uma confusão conceitual. Não por acaso, o autor sugeriu o abandono da distinção entre reforçamento positivo e reforçamento negativo no ensino da análise do comportamento. O presente trabalho teve como objetivo avaliar como os conceitos de reforçamento positivo e reforçamento negativo são abordados em livros de ensino de análise do comportamento. Foram utilizados 13 livros de ensino de análise do comportamento, sendo extraídos trechos que apresentam as definições dos conceitos mencionados. Os resultados apontam que apenas dois trabalhos de fato abordam os problemas relacionados aos conceitos de reforçamento positivo e reforçamento negativo. Conclui-se que o ensino da análise do comportamento tem ignorado os argumentos apresentados por Michael (1975) no ensino dos conceitos investigados. Aponta-se que essa investigação pode ser ampliada para investigar como os conceitos são tratados em publicações de artigos.
\end{abstract}

Palavras-chaves: reforçamento positivo, reforçamento negativo, ensino de análise do comportamento. 
Abstract: The concepts of positive reinforcement and negative reinforcement have been traditionally defined by the presentation of a reinforcing stimulus and by the removal of aversive stimuli, respectively. Michael (1975) questioned this distinction, stating that in many situations is difficult to distinguish such processes based on presentation and removal. Furthermore considered wrong the treatment as synonyms of negative reinforcing stimuli and aversive stimuli interchangeably, which identify negative reinforcement with punishment and create a conceptual confusion. Not by chance, he suggested the abandonment of the distinction between positive reinforcement and negative reinforcement in teaching behavior analysis. The present paper had the objective to evaluate how the concepts of positive reinforcement and negative reinforcement are treated in behavior analysis textbooks. 13 behavior analysis textbooks were used, being extracted transcriptions the present the definitions of the concepts mentioned above. The results showed that only two books dealt with the problems related to the concepts of positive reinforcement and negative reinforcement. We conclude that, when teaching behavior analysis, the Michael's (1975) arguments are ignored. This investigation could be enhanced by investigating how such concepts are treated in articles published in peer-reviewed journals.

Keywords: positive reinforcement, negative reinforcement, teaching Behavior Analysis

Resumen: Los conceptos de refuerzo positivo y refuerzo negativo se han definido tradicionalmente por la presentación de un estímulo de refuerzo y por la eliminación de estímulos aversivos, respectivamente. Michael (1975) cuestionó esa distinción, afirmando que en muchas situaciones es difícil distinguir estos procesos sobre la base de la apresentación y la eliminación. Además, consideró equivocado lo tratamiento como sinónimos de los estímulos refuerzadores negativos y estímulos aversivos, que identifican refuerzo negativo com castigo y crear una confusión conceptual. No es coincidencia que el autor sugirió el abandono de tal distinción en la enseñanza de análisis de la conducta. El presente trabajo tuvo el objetivo de evaluar cómo se tratan los conceptos de refuerzo positivo y refuerzo negativo en análisis de la conducta libros de texto. Se utilizaron análisis de comportamiento 13 libros de texto, que se extrae transcripciones presente las definiciones de los conceptos mencionados anteriormente. Los resultados mostraron que sólo dos libros tratan los problemas relacionados con los conceptos de refuerzo positivo y refuerzo negativo. Llegamos a la conclusión de que, en la enseñanza de análisis de la conducta, la confusión conceptual aquí mencionado ha tenido ignorado los argumentos de Michael (1975). Esta investigación podría mejorar la investigación de cómo estos conceptos se tratan en los artículos publicados en revistas revisadas por pares.

Palabras-clave: refuerzo positivo, refuerzo negativo, enseñanza de análisis de la conducta 
Uma característica inerente a toda modalidade científica é o desenvolvimento de conceitos descritivos/explicativos que permitam a análise do seu objeto de estudo. Quanto mais esses conceitos forem coerentes e interligados, maior será a qualidade das análises que os empreguem. No âmbito da análise do comportamento, conceitos básicos extensamente utilizados, como os conceitos de reforçamento positivo e reforçamento negativo, apresentam problemas que permitem aos analistas do comportamento questionar a necessidade de tal distinção (Michael, 1975).

O reforçamento positivo é geralmente definido como o fortalecimento de uma resposta devido à apresentação de determinado estímulo a ela contingente; já o reforçamento negativo consiste no aumento na frequência de uma resposta por causa da remoção contingente de um estímulo (Skinner, 1953/2007). Michael (1975) revisou esses conceitos, posicionando-se a favor do abandono de tal distinção. Além de alertar para o fato de que estímulos reforçadores negativos (aqueles que fortalecem respostas que os eliminam) podem ser confundidos com estímulos aversivos (aqueles que suprimem respostas que os produzem), o que poderia induzir a uma identificação entre reforçamento negativo e punição, Michael identificou uma segunda questão problemática no uso de tais conceitos: a ambiguidade inerente a essa distinção.

Considerando o primeiro problema, uma vez que a análise do comportamento adota um paradigma explicativo relacional, seus conceitos devem ser definidos também de forma relacional. Assim, o problema em identificar reforçadores negativos com estímulos aversivos poderia levar à conclusão de que um reforçador negativo apresenta efeitos supressores quando produzido por uma resposta, quando na verdade o termo reforçamento implica fortalecimento, não supressão (Michael, 1975). Segundo Catania (1998/1999), os estímulos não possuem propriedades fortalecedoras ou supressoras por si sós, mas adquirem tais funções na relação entre o responder do organismo e seus efeitos no ambiente. Quando um estímulo previamente identificado como reforçador negativo em uma dada relação demonstra propriedades supressoras em outra, seu rótulo deve ser modificado (no caso, pode-se descrevê-lo como punidor).
Catania (1998/1999) concorda que o critério de apresentação e de remoção de estímulos não deveria ser tão importante na distinção entre reforçamentos positivo e negativo. Pode-se ilustrar o problema da ambiguidade com o experimento de Weiss e Laties (1961), conforme citado por Catania (1998/1999, p. 120). Nesse experimento, foi utilizada uma câmara fria, na qual as pressões à barra por um rato eram consequenciadas com a operação de um aquecedor. Dessa forma, as pressões à barra eram reforçadas positivamente com a apresentação de energia ao ambiente. No entanto, também seria convincente a observação de que as pressões à barra removem o frio do ambiente, o que exemplificaria um caso de reforçamento negativo.

Esse problema também pode ser encontrado em diversos exemplos experimentais e da vida cotidiana que explicitam a ambiguidade de definir tais conceitos com base na apresentação ou na remoção de um estímulo: a apresentação de uma pelota de alimento para um animal privado pode ser interpretada como um caso de reforçamento positivo, mas também elimina a condição de privação; o recebimento de salário contingente ao mês trabalhado pode ter função de reforçador positivo quando o trabalhador chega ao fim do mês com sua conta bancária em saldo positivo, ou pode ter função de reforçador negativo quando o trabalhar chega ao fim do mês com sua conta em saldo negativo. Assim, a distinção entre reforçamentos positivo e negativo com base em apresentação e em remoção de estímulos pode ser considerada ambígua, visto que é possível identificar eventos adicionados e subtraídos do ambiente em ambos os casos.

Michael (1975) descreveu três possíveis razões para a existência dessa distinção, apesar de rejeitá-las. A primeira razão diz respeito ao fato de que o efeito fortalecedor no reforçamento positivo difere, de algum modo, do efeito fortalecedor no reforçamento negativo. Pode haver diferenças nas propriedades temporais, nas relações com outras variáveis independentes ou no papel que exercem no desenvolvimento de discriminações. Michael (1975) reconhece que as propriedades únicas dos reforçadores são similares entre reforçamento positivo e reforçamento negativo (ver Hineline, 1984). A magnitude do reforçador e a manipulação do tempo entre a emissão da resposta e a produção do 
reforçador, por exemplo, têm influências parecidas sobre o responder positiva e negativamente reforçados. Isso não significa que não existam diferenças. Baron e Galizio (2005) levantaram a hipótese de que a rapidez do fortalecimento é um fator diferente em ambas as formas de reforçamento - talvez a mudança de estímulo seja mais abrupta em casos de reforçamento negativo, justificando sua maior velocidade em fortalecer operantes. Essa interpretação ainda carece de validação empírica, mas é possível que, mesmo confirmada, não promova uma alteração no modo como é compreendido o processo de reforçamento.

A segunda razão indicada por Michael (1975) seria a existência de diferenças nas estruturas ou nos processos fisiológicos subjacentes aos reforçamentos positivo e negativo. A manutenção da distinção favoreceria ligações entre pesquisas comportamentais e fisiológicas, mas as pesquisas neurobiológicas e farmacológicas não têm produzido resultados úteis nesse sentido (Baron \& Galizio, 2005).

A terceira razão sugerida por Michael (1975) afirma que, por manter a distinção, seria mais fácil advertir sobre os efeitos indesejáveis do reforçamento negativo. Todavia, há efeitos que também poderiam ser considerados indesejáveis em casos de reforçamento positivo, como quando altos graus de privação são necessários para o estudo de determinado padrão de respostas (Baron \& Galizio, 2005). Além disso, se a própria distinção é confusa, não há como garantir a efetividade de avisos sobre efeitos indesejáveis.

As três possíveis razões para a manutenção dessa distinção, consideradas por Michael (1975), não esgotaram as possibilidades. Baron e Galizio (2005) identificaram outras três possíveis razões e teceram comentários sobre elas. A primeira delas refere-se aos sentimentos associados ao reforço. Skinner (1974/2006) sugeriu que diversos sentimentos são característicos de esquemas de reforço específicos. Poder-se-ia utilizar os sentimentos associados ao reforço como elemento-chave na distinção entre reforçamentos positivo e negativo, supondo que sejam sentimentos diferentes em cada situação. Geralmente, indivíduos expostos a esquemas de reforçamento positivo relatam sentir prazer ou satisfação. Por sua vez, em esquemas de reforçamento negativo, os relatos são mais próximos de alívio ou redução da dor e da ansiedade.

No caso do reforçamento positivo, os sentimentos são correlacionados às situações nas quais o estímulo reforçador está ausente; no caso do reforçamento negativo, aos próprios reforçadores negativos. Por exemplo, na produção de comida como reforçador, os sentimentos são evocados pela condição prévia de ausência de comida; já no caso da eliminação de choques, os sentimentos são evocados pelo próprio choque. Para Baron e Galizio (2005), os sentimentos dificilmente são úteis na distinção entre reforçamentos positivo e negativo. No estudo com humanos, o conhecimento da condição sentida dependerá em grande medida dos relatos verbais dos próprios indivíduos, os quais ocasionalmente são imprecisos. Um exemplo é a estimulação corporal de alta intensidade sentida como dor por uns indivíduos, mas como prazer por aqueles rotulados como masoquistas. Já nos estudos com animais não humanos, essa informação ainda não está disponível.

Uma segunda razão apresentada por Baron e Galizio (2005) é o papel das respostas competitivas. Ao abordar essa questão, conforme já mencionado, Catania (1998/1999) utilizou como exemplo o experimento conduzido por Weiss e Laties (1961). $\mathrm{Na}$ fuga do frio, pode-se argumentar que o reforço envolve tanto apresentação quanto remoção de um estímulo. Antes de pressionar a barra, o sujeito encolhia-se e tremia. Essas respostas competiam (i.e., reduziam a probabilidade) com a resposta de pressionar a barra. Quando o aquecedor era ligado, as respostas competitivas tornavam-se menos prováveis. Se as respostas competitivas ocorressem antes do reforço, o processo seria considerado reforçamento negativo. Se as respostas competitivas ocorressem após o reforço, o processo seria de reforçamento positivo. No entanto, Catania (1998/1999) e Baron e Galizio (2005) argumentam que esse critério não resolve a ambiguidade. Considerando um sujeito privado de alimento, a própria privação evoca respostas competitivas, como lamber ou inspecionar o comedouro. Além disso, há exemplos de comportamentos de esquiva na ausência de respostas competitivas (e.g., programar o despertador para acordar em determinado horário).

O terceiro motivo analisado por Baron e Galizio (2005) envolve as operações estabelecedo- 
ras e seu papel no controle do comportamento. A intensidade da operação estabelecedora exerce um papel central na diferença entre reforçamentos positivo e negativo. No caso do reforçamento positivo, um elevado grau de privação intensificaria os sentimentos associados ao reforço e a efetividade do reforçador. No caso do reforçamento negativo, por sua vez, a força do reforçador tem relação direta com a intensidade e com a duração do estímulo eliminado. Contudo, ainda não existe um modo claro de fazer uma escala de diferentes operações estabelecedoras.

Michael (2006) sugere que livros de ensino de análise do comportamento abordem a distinção entre reforçamentos positivo e negativo da maneira tradicional, mas que posteriormente comentem a ambiguidade dos conceitos, dando lugar a uma distinção mais clara entre processos de reforçamento e de punição, sem mencionar se ocorrem por apresentação ou por remoção.

Diante do exposto, o objetivo deste estudo foi analisar a distinção entre reforçamentos positivo e negativo encontrada em livros de ensino de análise do comportamento. Esta análise se justifica pela predominância da distinção tradicional sem que seja abordada a problemática envolvida no uso dos termos. Além disso, a literatura carece de uma revisão sistemática que investigue como os conceitos estão sendo ensinados.

\section{Método}

\section{Procedimento}

Foram utilizados 13 livros de ensino de análise do comportamento comumente adotados em cursos de graduação e de pós-graduação, em uma revisão bibliográfica com enfoque nos conceitos de reforçamento positivo e reforçamento negativo. A busca pelos capítulos mais relevantes dos livros foi feita por meio da sondagem de seus respectivos Sumários, e os capítulos selecionados foram aqueles que abordavam o tema reforçamento positivo e reforçamento negativo.

\section{Análise de Dados}

Os dados obtidos durante a revisão de literatura foram listados em uma tabela organizada de acordo com o autor, o título do livro, o capítulo do livro e a definição dos conceitos de reforçamento positivo e reforçamento negativo (ver Apêndice).

\section{Resultados e Discussão}

Os dados coletados nos livros de ensino apontam que existe concordância entre as descrições dos conceitos reforçamento positivo e reforçamento negativo. Todos os livros utilizam os termos positivo e negativo como sinônimos, respectivamente, de apresentação e remoção. Dessa forma, a ambiguidade faz-se presente nos livros analisados. As citações que incluem as descrições dos conceitos de reforçamento positivo e negativo podem ser encontradas no Apêndice deste artigo.

A apresentação dos resultados será dividida em textos publicados antes do artigo de Michael (1975) e textos publicados depois desse trabalho. A Tabela 1 apresenta as publicações analisadas e aponta aquelas que mostram a distinção tradicional entre reforçamentos positivo e negativo, bem como aquelas que consideram as críticas de Michael (1975) em suas definições.

\section{Tabela 1}

Publicações Analisadas quanto à Consideração ou Não das Críticas de Michael (1975)

\begin{tabular}{|c|c|c|}
\hline Publicação & $\begin{array}{l}\text { Apresenta a vi- } \\
\text { são tradicional }\end{array}$ & $\begin{array}{l}\text { Considera } \\
\text { a crítica de } \\
\text { Michael (1975) }\end{array}$ \\
\hline $\begin{array}{l}\text { Keller e Schoenfeld } \\
(1950 / 1974)\end{array}$ & $X$ & \\
\hline Skinner (1953/2007) & $x$ & \\
\hline Reese (1966/1973) & $x$ & \\
\hline $\begin{array}{l}\text { Deese e Hulse } \\
(1967 / 1975)\end{array}$ & $x$ & \\
\hline Millenson (1967/1975) & $x$ & \\
\hline $\begin{array}{l}\text { Holland e Skinner } \\
(1969 / 1975)\end{array}$ & $x$ & \\
\hline Lundin (1969/1972) & $x$ & \\
\hline Whaley e Malott (1981) & $x$ & \\
\hline Sidman (1989/1995) & $x$ & \\
\hline Baum (1994/1999) & $x$ & \\
\hline Pierce e Epling (1995) & $x$ & $x$ \\
\hline Catania (1998/1999) & $x$ & $x$ \\
\hline $\begin{array}{l}\text { Cameschi e Abreu- } \\
\text { Rodrigues (2005) }\end{array}$ & $x$ & $x$ \\
\hline
\end{tabular}




\section{Publicações Antes de Michael (1975)}

Entre as publicações, foram encontrados os trabalhos de Keller e Schoenfeld (1950/1974), Skinner (1953/2007), Reese (1966/1973), Deese e Hulse (1967/1975), Millenson (1967/1975), Lundin (1969/1972) e Holland e Skinner (1969/1975). Todos os autores definem reforçamento positivo e reforçamento negativo pela distinção tradicional, sem nenhuma crítica ao modelo e com descrições mais ou menos próximas. No entanto, alguns autores merecem considerações adicionais. Keller e Schoenfeld (1950/1974) definem reforço negativo tanto pelo efeito fortalecedor de sua remoção quanto pelo efeito enfraquecedor de sua apresentação. Como já mencionado, essa conceituação é problemática devido ao fato de que o termo reforçador é sinônimo de fortalecimento, e não de enfraquecimento (Michael, 1975). Dessa forma, é feita uma confusão entre os conceitos de punição e reforço negativo, conforme explicitada pela citação abaixo:

Uma outra maneira, e talvez melhor, de tratar o assunto é a de definir reforços positivos como aqueles estímulos que intensificam as respostas quando presentes (por exemplo, alimento intensifica o pressionar a barra ou o comportamento de puxar um cordão), e reforços negativos como aqueles que intensificam [as respostas] quando removidos. . . Temos, então, duas maneiras de definir reforços negativos: a primeira é em termos do efeito de enfraquecimento que têm quando apresentados; a segunda é em termos do efeito de reforçamento, pela sua remoção. O efeito se faz sentir sobre o comportamento operante; um operante é enfraquecido num caso e reforçado no outro. No entanto, deve-se notar que o mesmo operante não deve sofrer as duas modificações simultaneamente. (Keller \& Schoenfeld, 1950/1974, pp. 75-76)

Skinner (1953/2007) também trabalha com a distinção tradicional entre reforçamentos positivo e negativo, sendo que seu modelo de controle aversivo deriva do argumento de que a punição não é considerada um processo comportamental, mas um procedimento derivado do processo de reforçamento. Alguns estudiosos do controle aversivo, como Azrin e Holz (1966), discordam dessa posi- ção e apresentam dados que demonstraram que a punição possui efeitos supressores distintos, considerando-a um processo comportamental único. Desse modo, Skinner evita possíveis confusões entre processos de reforçamento negativo e punição, ao não qualificar este segundo como um processo comportamental básico.

Embora distinga entre os dois processos com base na apresentação e na remoção de estímulos, Lundin (1969/1972) utiliza o experimento realizado por Olds e Milner (1954), citado pelo próprio Lundin, no qual se investigou a produção de choques direcionados a diferentes áreas do cérebro, contingentes ao comportamento de pressão à barra. Os autores verificaram que, dependendo da área afetada, o comportamento de pressão à barra variava de 2000 até 5000 pressões por hora. Sendo assim, concluíram que existem áreas do cérebro relacionadas ao reforço positivo e ao reforço negativo. Adicionalmente, deve-se destacar que o trecho encontrado na obra de Lundin (1969/1972) para definir os conceitos é uma citação retirada de Keller e Schoenfeld (1950/1974).

Conforme esperado, pode ser observado que nenhuma das fontes pesquisadas publicadas antes do trabalho de Michael (1975) critica as distinções tradicionais entre os conceitos de reforçamento positivo e negativo.

\section{Publicações Posteriores a Michael (1975)}

Nas publicações posteriores à crítica de Michael (1975), foram encontrados trabalhos que tanto usam apenas as definições tradicionais quanto abordam sua crítica ao apresentar a distinção entre reforçamentos positivo e negativo. No primeiro grupo, foram alocados os trabalhos de Baum (1994/1999), Sidman (1989/1995) e Whaley e Malott (1981). Desses, a descrição de Baum (1994/1999) merece comentários especiais. O autor parte da definição tradicional e distingue reforços positivo e negativo utilizando expressões como "mais provável" e "menos provável" em substituição, respectivamente, à apresentação e à remoção. No entanto, Baum não menciona a problemática na distinção entre os conceitos. Além disso, sua própria definição, embora com outros termos, também revela ambiguidade. Afirmar que a apresentação de dinheiro torna-se mais provável quando contin- 
gente ao ato trabalhar é o mesmo que dizer que a remoção ou a diminuição de um estado de privação torna-se menos provável.

Pierce e Epling (1995), Catania (1998/1999) e Cameschi e Abreu-Rodrigues (2005) levam em consideração a crítica de Michael (1975) em suas descrições dos referidos conceitos. Catania (1998/1999) afirma que o critério de acréscimo ou remoção possui falhas. Cita o experimento de Weiss e Laties (1961) e analisa o argumento de que o momento no qual surgem as respostas competitivas é crucial para fazer a distinção. No entanto, acrescenta que o reforço sempre envolve mudanças no ambiente que conduzem a diferenças no responder antes e após a mudança, tornando inválido o argumento das respostas competitivas.

Pierce e Epling (1995) apresentam suas descrições com maior ênfase nas críticas aos conceitos. Os autores citam Michael (1975) ao comentarem a dificuldade em distinguir entre reforçamentos positivo e negativo. Destacam uma sugestão feita por Hineline (1984) de que existe uma assimetria fundamental entre os dois processos: no reforçamento positivo, o estímulo está ausente quando a resposta ocorre; já no reforçamento negativo, a estimulação a ser removida está presente. No entanto, esta sugestão parece não resolver o problema. No caso do reforçamento positivo, por exemplo, um sujeito privado de alimento produz pelotas de ração que não estavam presentes no ambiente. Ao mesmo tempo, pode-se considerar que este mesmo sujeito remove uma condição de privação presente antes de a resposta ocorrer. Além disso, há casos nos quais não se identifica uma condição aversiva presente que será removida quando o sujeito se comportar (e.g., ao programar um despertador que tocará na manhã seguinte).

Cameschi e Abreu-Rodrigues (2005) chegam a abordar a crítica do Michael (1975) apontando que seus questionamentos acerca da utilidade da distinção entre os conceitos de reforçamento positivo e negativo derivam, em parte, do fato de que o uso original desses conceitos visava a distinguir, respectivamente, entre os efeitos do reforço e os da punição. Apesar de apontarem essa crítica, os autores parecem aceitar a distinção comum de que positivo refere-se à produção de eventos e negativo, à remoção.

\section{Considerações Finais}

Este artigo teve como objetivo analisar a distinção entre reforços positivo e negativo, encontrada em livros de ensino de análise do comportamento. Os argumentos propostos por Michael (1975) para demonstrar a problemática envolvida nessa distinção, revisados e ampliados por Baron e Galizio (2005), embora não tenham sido refutados, parecem ser negligenciados no ensino da análise do comportamento.

Alguns autores têm identificado outros aspectos que favorecem a manutenção da distinção, embora Michael (1975) e Baron e Galizio (2005) defendam que a ambiguidade deva ser eliminada, definindo os eventos como reforçamento e punição. No entanto, fora do campo de livros de ensino de análise do comportamento, há autores que discutem aspectos que favorecem a manutenção da distinção (e.g., Chase, 2006; Iwata, 2006).

Chase (2006) concorda com os argumentos expostos por Baron e Galizio (2005), mas indica que talvez a distinção tenha uma função útil. Segundo ele, o ensino da análise do comportamento pode ser facilitado com a distinção entre reforçamentos positivo e negativo. Os exemplos desses casos permitem direcionar a atenção dos alunos para detalhes do tipo de variáveis que podem exercer controle sobre o comportamento.

Para Iwata (2006), a distinção pode ser útil desde que seja feita de forma cuidadosa. Embora problemática, ela descreve operações comportamentais que podem ser úteis na análise de uma variedade de casos. Ainda que concorde com Michael (1975), Iwata (2006) oferece os seguintes motivos para que a distinção seja mantida: (a) várias mudanças de estímulos envolvem condições relevantes antes e depois das mudanças nas quais é possível determinar quais estímulos estão presentes ou ausentes; (b) a identificação das diferenças críticas entre as condições antes e após as mudanças de estímulos facilita o desenvolvimento de contingências efetivas, o que tem grande importância para a área aplicada.

Apesar de ser alvo de controvérsias, com autores posicionando-se contra e a favor do abandono da distinção entre reforçamentos positivo e negativo, parece haver concordância a respeito da falha numa distinção puramente entre apresentação e 
remoção de estímulos. Michael (2006) comenta Baron e Galizio (2005) e conclui afirmando que seria interessante conhecer essa distinção, mas não incentivar seu uso. Como dito anteriormente, ele também sugere que livros didáticos podem iniciar descrevendo a distinção em seu sentido tradicional, mas deveriam optar por utilizar apenas a distinção entre reforçamento e punição no restante do livro.

Um complemento a esta revisão inicial seria investigar como artigos conceituais, experimentais e aplicados abordam os conceitos de reforçamento positivo e negativo. Talvez existam diferenças na utilização dos conceitos pelas áreas experimental e aplicada. Duas possibilidades de trabalhos averiguando os impactos das críticas aos conceitos podem ser: (a) uma tentativa de avaliar o impacto da publicação do artigo de Michael (1975) em publicações antes e depois do ano de 1975 e (b) uma tentativa de avaliar o impacto da publicação do artigo de Baron e Galizio (2005) em publicações antes e depois do ano de 2005.

Embora o presente estudo demonstre que algumas publicações posteriores ao trabalho de Michael (1975) levam sua crítica em consideração, boa parte dos livros de ensino de análise do comportamento mantém a distinção tradicional. Reconhece-se, assim, uma importância para essa distinção, mas é imprescindível que seja levantado o debate dos motivos pelos quais a crítica de Michael (1975) tem sido pouco considerada. Estudos posteriores poderiam trabalhar na direção de buscar entender a manutenção da definição tradicional como padrão no ensino de análise do comportamento. Estudos de revisão das literaturas experimental e aplicada poderiam discutir se a crítica apontada por Michael (1975) tem direcionado de fato investigações básicas, aplicadas e intervenções. Tal discussão poderia justificar possíveis motivos para manutenção da distinção tradicional. Pesquisas de história e/ ou sociologia da ciência no âmbito da análise do comportamento poderiam ainda apontar motivos relacionados a ligações entre grupos de pesquisa e indivíduos da comunidade analítico-comportamental que possibilitem um maior entendimento da relutância de nossa comunidade ao uso da crítica de Michael (1975). Desse modo, até que uma distinção mais parcimoniosa e coerente seja proposta, defendemos que os argumentos expostos por
Michael (1975), ampliados e revisados por outros autores, deveriam ser levados em consideração no ensino da análise do comportamento.

\section{Referências}

Azrin, N. H. \& Holz, W. C. (1966). Punishment. Em W. K. Honig (Org.), Operant behavior: Areas of research and application (pp. 380-447). New York: Appleton-Century-Crofts.

Baron, A. \& Galizio, M. (2005). Positive and negative reinforcement: Should the distinction be preserved? The Behavior Analyst, 28, 85-98.

Baum, W. M. (1999). Compreender o behaviorismo: Ciência, comportamento e cultura (M. T. A. Silva, M. A. Matos, G. Y. Tomanari \& E. Z. Tourinho, Trads.). Porto Alegre, RS: Artes Médicas. (Trabalho original publicado em 1994)

Cameschi, C. E. \& Abreu-Rodrigues, J. (2005). Contingências aversivas e comportamento emocional. Em J. Abreu-Rodrigues \& M. R. Ribeiro (Orgs.), Análise do comportamento: Teoria, pesquisa e aplicação (pp. 113-137). Porto Alegre, RS: Artmed.

Catania, A. C. (1999). Aprendizagem: Comportamento, linguagem e cognição (4a ed.; D. G. Souza, Trad.). (Porto Alegre, RS: Artmed. (Trabalho original publicado em 1998)

Chase, P. N. (2006). Teaching the distinction between positive and negative reinforcement. The Behavior Analyst, 29, 113-115.

Deese, J. \& Hulse, S. H. (1975). A psicologia da aprendizagem (D. M. Leite, Trad.). São Paulo, SP: Pioneira. (Trabalho original publicado em 1967).

Hineline, P. N. (1984). Aversive control: A separate domain? Journal of the Experimental Analysis of Behavior, 42, 495-509.

Holland, J. G. \& Skinner, B. F. (1975). A análise do Comportamento (R. Azzi \& C. M. Bori, Trads.). São Paulo, SP: Editora Pedagógica e Universitária. (Trabalho original publicado em 1969).

Iwata, B. A. (2006). On the distinction between positive and negative reinforcement. The Behavior Analyst, 29, 121-123.

Keller, F. S. \& Schoenfeld, W. N. (1974). Princípios de psicologia (C. M. Bori \& R. Azzi, Trads.). São 
Paulo, SP: Editora Pedagógica e Universitária. (Trabalho original publicado em 1950).

Lundin, R. W. (1972). Personalidade: Uma análise do comportamento (R. L. Rodrigues \& L. O. S. Queiroz, Trads.). São Paulo, SP: Herder. (Trabalho original publicado em 1969)

Michael, J. (1975). Positive and negative reinforcement: A distinction that is no longer necessary; or a better way to talk about bad things. Behaviorism, 3, 33-44.

Michael, J. (2006). Comment on Baron and Galizio (2005). The Behavior Analyst, 29, 117-119.

Millenson, J. R. (1975). Princípios de análise do comportamento (A. A. Rosa \& D. Resende, Trads.). Brasília, DF: Editora de Brasília. (Trabalho original publicado em 1967)

Pierce, W. D. \& Epling, W. F. (1995). Behavior analysis and learning. Englewood Cliffs, NJ: Prentice-Hall.
Reese, E. P. (1973). Análise do comportamento humano (G. P. Witter, Trad.). Rio de Janeiro, RJ: Livraria José Olympio. (Trabalho original publicado em 1966)

Sidman, M. (1995). Coerção e suas implicações (M. A. Andery \& T. M. Sério, Trads.). Campinas, SP: Psy. (Trabalho original publicado em 1989)

Skinner, B. F. (2006). Sobre o behaviorismo. São Paulo, SP: Cultrix. (Trabalho original publicado em 1974)

Skinner, B. F. (2007). Ciência e comportamento humano (J. C. Todorov \& R. Azzi, Trads.). São Paulo, SP: Martins Fontes. (Trabalho original publicado em 1953).

Whaley, D. L. \& Malott, R. W. (1981). Princípios elementares do comportamento (M. A. Matos, M. L. F. \& C. F. Santoro, Trads.). São Paulo, SP: Editora Pedagógica e Universitária.

Weiss, B. \& Laties, V. G. (1961) Behavioral Thermoregulation. Science, 133, 1338-1344.

\section{Apêndice}

Tabela 2

Apresentação dos conceitos de reforçamento positivo e negativo segundo seus respectivos autores.

\begin{tabular}{|c|c|c|}
\hline Autor/Ano & Título/Capítulo do livro & Conceituação \\
\hline $\begin{array}{l}\text { Keller e } \\
\text { Schoenfeld } \\
(1950 / 1974)\end{array}$ & $\begin{array}{l}\text { Condicionamento operan- } \\
\text { te (capítulo 3) }\end{array}$ & $\begin{array}{l}\text { "Uma outra maneira, e talvez melhor, de tratar o assunto é a de definir reforços } \\
\text { positivos como aqueles estímulos que intensificam as respostas quando presentes } \\
\text { (por exemplo, alimento intensifica o pressionar a barra ou o comportamento de } \\
\text { puxar um cordão), e reforços negativos como aqueles que intensificam quando } \\
\text { removidos. [...] Temos, então, duas maneiras de definir reforços negativos: a } \\
\text { primeira é em termos do efeito de enfraquecimento que tem quando apresentados; } \\
\text { a segunda é em termos do efeito de reforçamento, pela sua remoção. O efeito se } \\
\text { faz sentir sobre o comportamento operante; um operante é enfraquecido num caso } \\
\text { e reforçado no outro. No entanto deve-se notar que o mesmo operante não deve } \\
\text { sofrer as duas modificações simultaneamente." (p. 75-76) }\end{array}$ \\
\hline $\begin{array}{l}\text { Skinner } \\
(1953 / 2007)\end{array}$ & $\begin{array}{l}\text { O comportamento ope- } \\
\text { rante (capítulo 5) }\end{array}$ & $\begin{array}{l}\text { "Os eventos que se verifica serem reforçadores são de dois tipos. Alguns reforços } \\
\text { consistem na apresentação de estímulos, no acréscimo de alguma coisa, por } \\
\text { exemplo, alimento, água ou contato sexual - à situação. Estes são denominados } \\
\text { reforços positivos. Outros consistem na remoção de alguma coisa - por exemplo, } \\
\text { de muito barulho, de uma luz muito brilhante, de calor ou frio extremos, ou de um } \\
\text { choque elétrico - da situação. Estes se denominam reforços negativos. Em ambos } \\
\text { os casos o efeito do reforço é o mesmo: a probabilidade da resposta será aumen- } \\
\text { tada." }\end{array}$ \\
\hline $\begin{array}{l}\text { Reese } \\
(1966 / 1973)\end{array}$ & $\begin{array}{l}\text { Métodos de controle do } \\
\text { comportamento } \\
\text { (capítulo 3) }\end{array}$ & $\begin{array}{l}\text { "O comportamento operante é fortalecido pela apresentação do estímulo reforça- } \\
\text { dor (às vezes chamado de controle positivo) e também pela remoção do estímulo } \\
\text { aversivo (às vezes chamado de controle negativo). A remoção de um estímulo } \\
\text { aversivo contingente a uma resposta é chamada de reforçamento negativo." }\end{array}$ \\
\hline
\end{tabular}


Deese e Hulse (1967/1975)

Reforço e aprendizagem - princípios básicos (capítulo 2)

Millenson

$(1967 / 1975)$

Contingências aversivas (capítulo 17)

Holland e

Skinner

(1969/1975)

Lundin

(1969/1972)

Whaley e

Malott (1981)

Fuga e esquiva (capítulo 20)

(1989/1995)

Baum

(1994/1999)

Teoria da evolução e reforço (capítulo 4) (capítulo 2) ção (capítulo 3)

Nem todo controle é coerção (capítulo 2)
Sidman

"O conceito de reforço positivo fica suficientemente claro a partir da discussão anterior e da definição geral de reforço que acabamos de dar. Em termos comuns, um reforço positivo é um prêmio - que damos ao organismo depois de ter feito alguma coisa que desejamos que aprenda a fazer. Mas o que dizer de reforços negativos? [...] Os reforços negativos são os estímulos que fortalecem uma resposta quando são afastados se a resposta ocorrer."

"Como a operação que define esses eventos como reforçadores (sua remoção) é oposta, em caráter, àquela dos reforçadores positivos (definidos por sua apresentação), eles são conhecidos como reforçadores negativos. Em geral, reforçadores negativos constituem-se daqueles eventos cujo término (ou redução na intensidade) fortalecerá e manterá operantes." (p. 383)

Condicionamento operante: conceitos elementares

"Desligar a televisão durante um anúncio é reforçado pela supressão de um reforço positivo; ligar a televisão para um programa muito interessante é reforçado pela apresentação de um reforço negativo" (p. 52)

Condicionamento e extin-

"Reforçamento consiste aqui simplesmente na apresentação de algum estímulo quando a resposta é emitida. Se, como resultado, observamos que a frequência aumenta, designamos o estímulo como reforçador positivo." (Keller \& Schoenfeld, 1950 citado por Lundin, 1969/1972)

"A frequência de uma resposta específica será aumentada se esta resposta for regularmente seguida pela cessação da estimulação ou término da perda de reforçadores positivos. Tal procedimento é chamado de reforçamento negativo. [...] Os dois procedimentos diferem pelo fato de o reforçamento positivo envolver a apresentação de um evento ou privilégio, enquanto o reforçamento negativo envolve a remoção de eventos classificados usualmente como 'aversivos'". (p.188)

"No reforçamento positivo, a ação de uma pessoa é seguida pela adição, produção ou aparecimento de algo novo, algo que não estava lá antes do ato. No reforçamento negativo, uma ação subtrai, remove ou elimina algo, fazendo com que alguma condição ou coisa que estava lá antes do ato desaparecesse." (p. 55)

"A dependência entre trabalho e alimento é um exemplo de reforço positivo: reforço porque a relação tende a fortalecer ou a manter a ação (trabalhar) e positivo porque a ação torna provável o reforçador (alimento). A ação entre escovar os dentes e desenvolver cáries é um exemplo de reforço negativo: reforço porque a relação tende a manter a escovação dos dentes (a ação), e negativo porque escovar torna a cárie (o punidor) menos provável" (p. 77)

"Quando uma resposta resulta na apresentação de um estímulo reforçador, a

Pierce e Epling Reforçamento e extinção

(1995) do comportamento operante (capítulo 4) e Regulação aversiva do comportamento (capítulo 9)

contingência é chamada reforçamento positivo. [...] Quando uma resposta resulta na remoção de um evento aversivo, a contingência é chamada reforçamento negativo." (pp. 92-94) [...] "Na vida diária, a distinção entre reforçamento positivo e negativo é ocasionalmente incerta (Michael, 1975)." (p. 244)

Catania

$(1998 / 1999)$

As consequências do res"Quando uma resposta termina ou evita um estímulo aversivo e, assim, torna-se mais provável, o estímulo é chamado de reforço negativo. A distinção entre reforço positivo e reforço negativo depende se uma resposta produz ou remove um estímulo. Mais tarde encontraremos alguns problemas na terminologia do reforço positivo e negativo". (p. 117)

Cameschi e Abreuponder: controle aversivo (capítulo 6)

Contingências aversivas e "Michael (1975) questionou a utilidade da distinção entre reforçamento positivo e comportamento emocional (capítulo 7) negativo" [...] "A análise funcional estabelece que o termo positivo descreve uma relação de dependência ou contingência entre uma resposta e a produção de estímulos, e negativo refere-se à contingência entre uma resposta e a remoção de estímulos (p. 120)
Informações do Artigo

Histórico do artigo:

Submetido em: 24/09/2013

Primeira decisão editorial: 16/05/2014

Aceito em: 05/08/2014 